\title{
O029: Reporting and case management of bloodborne pathogen exposures among health care workers in Tanzania
}

\author{
M Lahuerta ${ }^{1,2^{*}}$, D Selenic ${ }^{3}$, G Mwakitosha ${ }^{1}$, J Hokororo ${ }^{4}$, H Ngonyani ${ }^{4}$, G Kassa ${ }^{1}$, R Mbatia ${ }^{5}$, SV Basavaraju ${ }^{3}$,

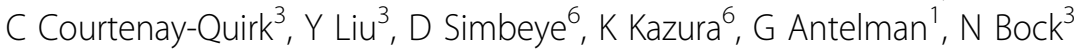

From 2nd International Conference on Prevention and Infection Control (ICPIC 2013)

Geneva, Switzerland. 25-28 June 2013

\section{Introduction}

In sub-Saharan Africa, bloodborne pathogens exposure (BPE) is a serious risk to health care workers (HCW). Reporting BPE is necessary for effective post-exposure prophylaxis (PEP), an important element of workplace safety in health facilities. Limited data are available on factors associated with BPE reporting among HCW.

\section{Methods}

We conducted a cross-sectional study assessing experiences of occupational BPE, history of BPE reporting, and use of PEP among health care workers at three public hospitals in Tanzania. From August to November 2012, HCW were interviewed using Audio-Computer Assisted Self-Interview. All HCW at risk for BPE were invited to participate. Factors associated with reporting BPE were identified using logistic regression.

\section{Results}

Of the 1,102 eligible HCW, 973 (88\%) completed the interview. Of these, 690 (71\%) were female and 387 (40\%) were nurses. Of $357 \mathrm{HCW}$ who had a BPE in the past 6 months, 120 (34\%) reported it. Among these 120 reported exposures, 93 (78\%) HCW reported within 2 hours of exposure, 98 (82\%) received pre- and post-HIV test counseling, and 70 (58\%) were offered PEP; 68 (97\%) of these $70 \mathrm{HCWs}$ completed PEP. Independent risk factors associated with reporting BPE were being female (adjusted odds ratio (AOR) $=2.0[95 \%$ confidence interval (CI) 1.2-3.5), having ever-received BPE training $(\mathrm{AOR}=2.0, \mathrm{CI} 1.2-3.5)$, knowledge that $\mathrm{HCW}$ receive
PEP at another facility (AOR=2.6, CI 1.5-4.4) and HIV testing within the past year (AOR=2.3, CI 1.2-4.4).

\section{Conclusion}

Despite the significant proportion of $\mathrm{HCW}$ with a recent $\mathrm{BPE}$, only one in three reported it. Our results highlight the importance of appropriate and continuous training on the prevention and reporting of occupational exposures to increase acceptance of HIV testing after BPE.

\section{Disclosure of interest}

None declared.

\section{Author details}

${ }^{1}$ ICAP-Columbia University, Mailman School of Public Health, USA

${ }^{2}$ Department of Epidemiology, Mailman School of Public Health, Columbia University, New York, USA. ${ }^{3}$ Divisions of Global HIV/AIDS, Centers for Disease Control and Prevention, Atlanta, USA. ${ }^{4}$ Ministry of Health and Social Welfare, Dar es Salaam, Tanzania, United Republic of. ${ }^{5}$ Tanzania Health Promotion Support (THPS), Dar es Salaam, Tanzania, United Republic of. ${ }^{6}$ US Centers for Disease Control and Prevention, Dar es Salaam, Tanzania, United Republic of.

Published: 20 June 2013

doi:10.1186/2047-2994-2-S1-029

Cite this article as: Lahuerta et al:: O029: Reporting and case management of bloodborne pathogen exposures among health care workers in Tanzania. Antimicrobial Resistance and Infection Control 20132 (Suppl 1):O29. 